Article

\title{
Indium Incorporation into InGaN Quantum Wells Grown on GaN Narrow Stripes
}

\author{
Marcin Sarzyński 1,2,*®D, Ewa Grzanka 1,2, Szymon Grzanka 1,2, Grzegorz Targowski 1,2, \\ Robert Czernecki ${ }^{1,2}$, Anna Reszka ${ }^{3}$, Vaclav Holy ${ }^{4}{ }^{\oplus}$, Shugo Nitta ${ }^{5}$, Zhibin Liu ${ }^{6}$, \\ Hiroshi Amano ${ }^{5,7,8}$ and Mike Leszczyński 1,2 \\ 1 Institute of High Pressure Physics PAS, Sokołowska 29/37, 01-142 Warsaw, Poland \\ 2 TopGaN Ltd., Sokołowska 29/37, 01-142 Warsaw, Poland \\ 3 Institute of Physics PAS, Al. Lotnikow 32/46, 02-668 Warsaw, Poland \\ 4 Faculty of Mathematics and Physics, Charles University, Ke Karlovu 5, 12116 Praha 2, Czech Republic \\ 5 Institute of Materials and Systems for Sustainability, Nagoya University, Furo-cho, Chikusa-ku, \\ Nagoya 464-8603, Japan \\ 6 Department of Electrical Engineering and Computer Science, Nagoya University, Furo-cho, Chikusa-ku, \\ Nagoya 464-8603, Japan \\ 7 Akasaki Research Center, Nagoya University, Furo-cho, Chikusa-ku, Nagoya 464-8603, Japan \\ 8 Venture Business Laboratory, Nagoya University, Furo-cho, Chikusa-ku, Nagoya 464-8603, Japan \\ * Correspondence: sarzyn@unipress.waw.pl; Tel.: +48-501-717-528
}

Received: 3 July 2019; Accepted: 6 August 2019; Published: 14 August 2019

check for updates

\begin{abstract}
InGaN quantum wells were grown using metalorganic chemical vapor phase epitaxy (vertical and horizontal types of reactors) on stripes made on GaN substrate. The stripe width was 5 , $10,20,50$, and $100 \mu \mathrm{m}$ and their height was 4 and $1 \mu \mathrm{m}$. InGaN wells grown on stripes made in the direction perpendicular to the off-cut had a rough morphology and, therefore, this azimuth of stripes was not further explored. InGaN wells grown on the stripes made in the direction parallel to the GaN substrate off-cut had a step-flow-like morphology. For these samples (grown at low temperatures), we found out that the InGaN growth rate was higher for the narrower stripes. The higher growth rate induces a higher indium incorporation and a longer wavelength emission in photoluminescence measurements. This phenomenon is very clear for the $4 \mu \mathrm{m}$ high stripes and less pronounced for the shallower $1 \mu \mathrm{m}$ high stripes. The dependence of the emission wavelength on the stripe width paves a way to multicolor emitters.
\end{abstract}

Keywords: InGaN; vapor phase epitaxy; patterned substrate; quantum wells; multicolor emitters

\section{Introduction}

Most electronic and optoelectronic devices are fabricated on laterally homogeneous epitaxial structures, however, having structures of properties varying in lateral directions would offer new possibilities, for example, monolithic integration of different devices. Such epitaxial structures are prepared by lateral patterning (lithography and masking) and overgrowth.

The first epitaxial lateral overgrowth (ELOG) of silicon and GaAs over SiOx masks was demonstrated almost 40 years ago [1-4]. This technology was then used for GaN growth on highly mismatched substrates-sapphire [5-7], SiC [8], or silicon [9]. In all cases, laterally grown GaN over the mask had substantially lower threading dislocation density than in areas of mask openings. The next step of this lateral overgrowth was pendeo epitaxy on stripes made on the GaN layer on foreign substrate [10]. Also, in that kind of epitaxy, the suspended wings contained much lower dislocations.

Pendeo epitaxy of GaN paved the way to growth of AlGaInN epitaxial structures on laterally pattered substrates. In the case of highly mismatched materials, lateral patterning may not only lead 
to lateral chemical composition variation, but also to elastic strain relaxation. Having submicron patterning, one may obtain quantum dots and wires [11,12].

In the present paper we focus on the growth of the ternary alloy, InGaN, on patterned GaN substrates. InGaN is the key material in III-Nitride optoelectronic devices such as light emitting diodes (LEDs) and laser diodes. operating in the blue and green ranges of the electromagnetic spectrum. Single and multiple quantum wells (SQWs, MQWs or just QWs for short) made of InGaN usually form the active region of such devices, which are typically grown on different sorts of $\mathrm{GaN}$ substrates. However, InGaN is relatively difficult to be grown high-quality (compared to the base binary compound, $\mathrm{GaN}$ ) because of its large lattice mismatch to $\mathrm{GaN} 11 \%[13,14]$ and low growth temperature- $700-800{ }^{\circ} \mathrm{C}$ for InGaN [15-17] versus $950-1050{ }^{\circ} \mathrm{C}$ for GaN. These two factors induce a number of crystallographic defects, such as indium concentration fluctuations [18-21] and a large concentration of point defects [22]. Such defects, in turn, adversely affect optical properties of devices.

Growth of InGaN on three-dimensional structures has been studied in a number of papers [23,24]. These papers dealt mainly with the growth of nanocolumns. Such three-dimensional growth is possible due to the low atomic incorporation at the side walls, and a high incorporation on the (00.1) surface. InGaN growth on a substrate with stripes was studied by Fang and coworkers [25] who studied the mass transport mechanism in InGaN epitaxy on ridge-shaped selective area growth GaN by metal organic chemical vapor deposition. In that work, however, emphasis was put on different aspects and the authors did not describe indium composition as a function of the stripe width. They also used selective mask for the growth, which is not the case in the present paper.

In one of our previous papers [26] we reported on InGaN quantum wells grown on stripes with various off-cuts. As indium incorporation into InGaN depends on the off-cut [27], by varying the off-cut spatially we could produce monolithically integrated multicolor emitters. However, when decreasing the size of the stripes we found out that not only the substrate off-cut, but also the size of the stripes influences indium incorporation into InGaN. The present paper reports on this phenomenon-we will show that the growth rate depends on the geometric dimensions of the stripes (width and height). Because indium incorporation into InGaN depends on the growth rate [28,29], consequently. In content also changes with the width of the stripes.

\section{Experimental}

\subsection{Substrate Preparation}

We used freestanding, c-plane (0001) GaN substrates from Saint-Gobain Lumilog company (Vallauris, France). Dislocation density was about $10^{7} / \mathrm{cm}^{2}$ and the off-cut angle was $0.6 \pm 0.05^{\circ}$ towards the m-direction $<1-100>$. Laser-beam photolithography (Microtech LW405B, Palermo, Italy) and ion etching with chlorine chemistry (Oxford Plasma Lab 100, Yatton, UK) were used to define the substrate pattern. The substrate scheme is shown in Figure 1a,b.

All prepared GaN substrates $(11 \times 14 \mathrm{~mm}$ in size) had five patterned regions marked P5, P10, P20, P50, and P100, each with size $1.5 \times 8 \mathrm{~mm}$. Stripe width, $w$, was 5, 10, 20, 50, and $100 \mu \mathrm{m}$ in regions P5, P10, P20, P50, and P100, respectively, as shown in Figure 1a,b. In each region, stripe separation (g) was equal to the respective stripe width (w), as shown in Figure 1a. Between regions P50 and P100 there was a flat $1000 \mu \mathrm{m}$ wide reference region without any stripes. Stripe direction was chosen along the m-direction $\langle 1-100\rangle$ as to be sure that during the epitaxial growth atomic steps could flow along the top of the stripe. However, we also prepared substrate with stripes perpendicular to the m-direction, as shown in Table 1. To check the morphology of QWs grown this way, this sample will be described in Section 3.1. Typically, the pattern consisted of a set of $4 \mu \mathrm{m}$ high parallel stripes. However, to check how luminescence depends on the height of the stripes we also prepared substrate with $1 \mu \mathrm{m}$ high stripes, as shown in Table 1, and results are described in Section 3.5. Before epitaxy, all substrates were cleaned in organic solvents and piranha. 


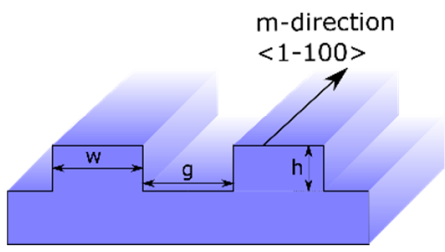

(a)

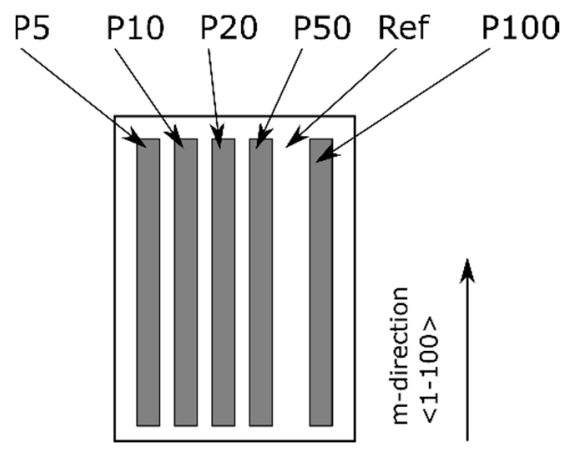

(b)

Figure 1. Stripes fabricated in GaN substrates. (a) Schematic perspective view and definition of stripe dimensions. (b) Scheme of a $11 \times 14 \mathrm{~mm}$ c-plane (0001) GaN substrate with five patterned regions marked P5, P10, P20, P50, and P100. The stripe width (w) is 5, 10, 20, 50, and $100 \mu \mathrm{m}$ in regions P5, P10, $\mathrm{P} 20, \mathrm{P} 50$, and P100, respectively. In all regions the gap between stripes ( $\mathrm{g}$ ) was equal to the stripe width $(\mathrm{g}=\mathrm{w})$. Stripe height $(\mathrm{h})$ was $4 \mu \mathrm{m}$ in standard samples and $1 \mu \mathrm{m}$ in all regions in the testing sample. The flat area named "Ref" of $1000 \mu \mathrm{m}$ wide, without stripes, was made as a reference. Each region P has dimensions $1.5 \times 8 \mathrm{~mm}$ and typically is fabricated along the m-direction.

Table 1. List of substrates. Symbols \| and $\perp$ mean "parallel to" and "perpendicular to", respectively.

\begin{tabular}{ccc}
\hline Substrate & $\mathbf{d}(\boldsymbol{\mu m})$ & Azimuth 3 \\
\hline 1 & 4 & $\| \mathrm{m}$ \\
2 & 4 & $\perp \mathrm{m}$ \\
3 & 1 & $\| \mathrm{m}$ \\
\hline
\end{tabular}

\subsection{Epitaxy Method}

The sidewall angle of patterned substrates before the growth was about $70-80^{\circ}$ with respect to the (0001) plane. Then, InGaN/GaN structures were grown on them, using metalorganic vapor phase epitaxy (MOVPE). The growth processes were carried out on two MOVPE reactors-one of them was a home-built, vertical reactor [30] located in the Institute of High Pressure Physics, Warsaw, Poland (IHPP) and the other was a commercial Taiyo Nippon-Sanso horizontal reactor [31] located in the Amano Lab, Institute of Materials and Systems for Sustainability, Nagoya University, Nagoya, Japan (IMaSS). We used two different reactors to confirm that the observed effect was not specific to a particular epitaxy system. The epitaxial structure grown in the above mentioned reactors consisted of subsequent layers: $0.5 \mu \mathrm{m} \mathrm{GaN}: S i$ (growth temperature $\mathrm{T}_{\text {gr }}=1040{ }^{\circ} \mathrm{C}$ ), $170 \mathrm{~nm}$ undoped $\operatorname{In}_{0.03} \mathrm{Ga}_{0.95} \mathrm{~N}$ buffer layer $\left(\mathrm{T}_{\mathrm{gr}}=810^{\circ} \mathrm{C}\right)$, and a single pair of undoped, $2 \mathrm{~nm}$ thick $\operatorname{In}_{0.25} \mathrm{Ga}_{0.75} \mathrm{~N}$ quantum well $(\mathrm{QW}) / 8$ $\mathrm{nm}$ GaN quantum barrier $(\mathrm{QB})\left(\mathrm{T}_{\mathrm{gr}}=760^{\circ} \mathrm{C}\right)$. An identical structure was grown on substrates with stripes in both directions, i.e., parallel and perpendicular to the m-direction (see Section 3.1). For the purpose of HR-XRD examination, a similar structure was grown on substrates with stripes parallel to the m-direction using the vertical reactor. It contained $5 \mathrm{QB} / \mathrm{QW}$ pairs (thicknesses 2 and $8 \mathrm{~nm}$, respectively) instead of one to improve the ability to track QW properties with X-ray methods-results are described in Section 3.3.

Efforts were made to carefully tune growth recipes for both reactors, to obtain identical structures. However, in MOVPE, indium composition is always very sensitive to many parameters, mainly growth temperature. For this reason, average indium composition achieved in the horizontal reactor was lower than for the vertical one. Nevertheless, the main effect was observed for structures grown in both reactors. 


\subsection{Sample Characterization}

Morphology of samples was checked by tapping mode atomic force microscope (AFM, Veeco Dimension 3100, Plainview, NY, USA).

Optical properties were studied at room temperature using two methods-micro-photoluminescence $(\mu \mathrm{PL})$ and cathodoluminescence (CL). In the $\mu \mathrm{PL}$ setup, luminescence was excited by a Kimmon He-Cd, continuous wave, $325 \mathrm{~nm}$ laser with $15 \mathrm{~mW}$ output power, and spectra were acquired by a iHR 320 spectrometer (Horiba Scientific, Piscataway, NJ, USA). Excitation spot size in this system was about $5 \mu \mathrm{m}$. CL measurements were performed with a SU-70 scanning electron microscope (Hitachi High Technologies, Tokyo, Japan) equipped with the Gatan Mono CL3 system (Pleasanton, CA, USA). Accelerating voltage ranged from 5 to $15 \mathrm{kV}$ and the beam current from 2.4 to $14 \mathrm{nA}$.

Structural properties were evaluated on the sample with 5 QW/QB pairs to increase signal intensity from QWs/QBs and improve ability to calculate indium content and width of QWs and QBs. The HR-XRD system (Empyrean-Malvern Panalytical, Almelo, The Netherlands) with a $\mathrm{CuK} \alpha_{1}$ X-ray source and equipped with hybrid 2-bounce monochromator and a threefold Ge (220) analyzer was used.

The structure grown in the horizontal reactor was investigated by scanning transmission electron microscopy (STEM) in high-angle annular dark-field (HAADF) operation mode, using Tecnai G2 F20 S-TWIN microscope (FEI Company, Hillsboro, OR, USA) operated at $200 \mathrm{kV}$. A cross-sectional STEM specimen was prepared by mechanical polishing and subsequent $\mathrm{Ar}+$ final ion milling until electron transparency using a Veraion precision ion polishing system (Gatan, Pleasanton, CA, USA).

Additionally, we examined the structure grown on patterned substrate with $4 \mu \mathrm{m}$ high stripes with a Dektak 150 stylus profiler (Veeco, Plainview, NY, USA).

\section{Experimental Results}

\subsection{Influence of Stripe Azimuth on the InGaN Morphology}

All structures were grown in the step-flow mode. The structure morphology on substrates with stripes parallel to the m-direction were similar to each other, both grown using vertical and horizontal MOVPE reactors. AFM images for structure grown using a vertical reactor is shown in Figure 2a,b. The morphology of the same structure grown on substrate with stripes perpendicular to the m-direction, i.e., along a-direction, was different and is shown in Figure 2c.

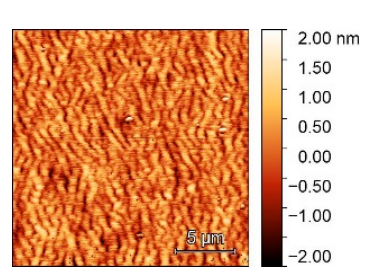

(a)

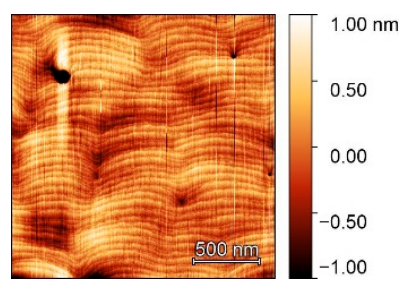

(b)

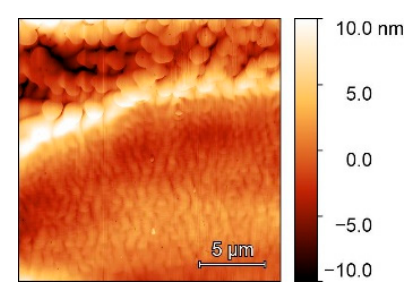

(c)

Figure 2. Atomic force microscope (AFM) height images of an InGaN/GaN structure grown on two substrates with stripes. In $(\mathbf{a}, \mathbf{b})$, stripe direction was parallel to substrate miscut azimuth and in (c) it was perpendicular.

The surface roughness (RMS) of QW grown on stripes parallel to the m-direction and measured by AFM on a $20 \times 20 \mu \mathrm{m}$ area was $0.52 \mathrm{~nm}$ while RMS of the QW grown on substrate with stripes perpendicular to the m-direction was $3.2 \mathrm{~nm}$.

These differences in layer morphology correspond to the directions of the stripe with respect to the atomic step direction of the substrate. For the substrates with stripes parallel to the m-direction, atomic steps were perpendicular to the stripe boundary and during growth they could flow along the stripe top without disturbances, resulting in a smooth surface, as shown in Figure $2 a, b$. On the 
contrary, for the substrate with stripes perpendicular to the m-direction, atomic steps were parallel to the stripe boundary and during growth they flew across the stripe, as shown in Figure 2c. Propagation length of an atomic step during growth, $l$, can be estimated as $l=d / \tan \delta$ where $d$ is layer thickness and $\delta$ is the substrate miscut. In our case, $d=0.68 \mu \mathrm{m}$ and $\delta=0.6^{\circ}$, so $l=65 \mu \mathrm{m}$ which is similar or larger than the stripe width. As a result, a large portion of the layer grew in an island mode due to the lack of source of atomic steps. The morphology of the InGaN QWs in the case of the stripes perpendicular to the m-direction was not acceptable due to large and non-uniform roughness; all further results are only for the QWs grown on substrates with stripes parallel to the m-direction.

\subsection{Influence of Stripe width on Luminescence of In $\mathrm{GaN} Q W$}

The most important result in the present paper is that luminescence wavelength of an InGaN QW/QB structure strongly increased when it was grown on a narrower stripe. More precisely, for single QW grown using a vertical reactor, $\mu$ PL wavelength shifted from 461 up to $495 \mathrm{~nm}$, and for stripe width variation, from 100 down to $5 \mu \mathrm{m}$, respectively. For nominally the same structure grown using a horizontal reactor, a similar effect was found, and $\mu \mathrm{PL}$ wavelength increased from 434 up to $451 \mathrm{~nm}$, and for stripe width change, from 100 to $5 \mu \mathrm{m}$, respectively. In both cases $\mu \mathrm{PL}$ was measured on top of each stripe, in the middle between stripe edges at room temperature. Luminescence wavelength as a function of stripe width is shown in Figure $3 a$ and $\mu \mathrm{PL}$ spectra are shown in Figure $3 b, c$.

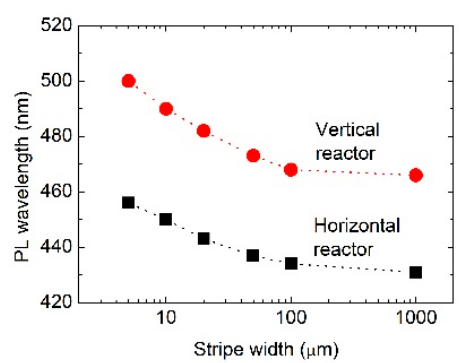

(a)

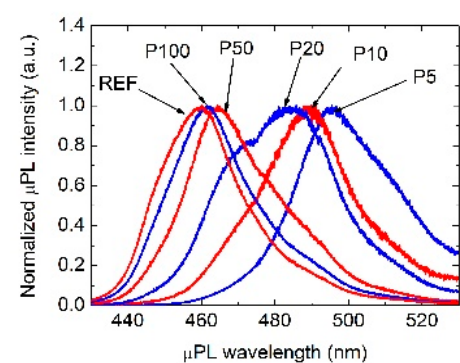

(b)

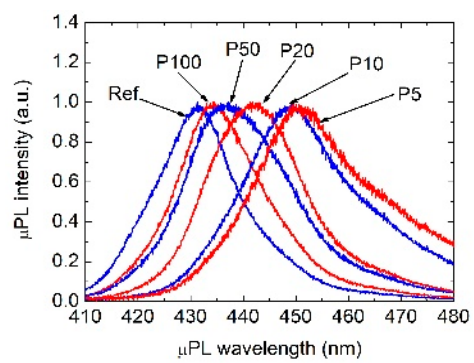

(c)

Figure 3. Micro-photoluminescence ( $\mu \mathrm{PL}$ ) of nominally identical InGaN/GaN structures grown on substrates with $4 \mu \mathrm{m}$ high stripes using vertical and horizontal reactors. (a) Central wavelength of $\mu \mathrm{PL}$ as a function of stripe width, (b) normalized $\mu \mathrm{PL}$ spectra of structure grown using a vertical reactor, (c) normalized $\mu \mathrm{PL}$ spectra of structure grown using a horizontal reactor.

In the next step we studied optical properties of our structure with single QW grown using a vertical, and also a horizontal reactor, in more detail. For both samples we performed $\mu$ PL scans along and across stripes in all regions P5-P100, as shown in Figure 4a. We also made CL measurements in three spots on top of the $50 \mu \mathrm{m}$ wide stripe-in the center, $5 \mu \mathrm{m}$ from the edge, and at the edge, as shown in Figure 5a. The $\mu \mathrm{PL}$ measurement scheme and results (for $100 \mu \mathrm{m}$ wide stripe) are shown in Figure 4. The CL measurement scheme and results (for $50 \mu \mathrm{m}$ wide stripe) are shown in Figure 4. It turned out that central $\mu \mathrm{PL}$ wavelength is constant along the stripe. However, central luminescence wavelength and full-width at half maximum (FWHM) of $\mu \mathrm{PL}$ and CL spectra increased from the center of each stripe towards its edges. 


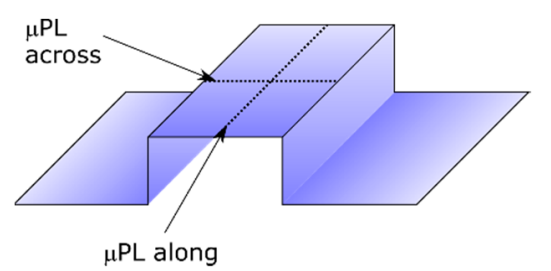

(a)

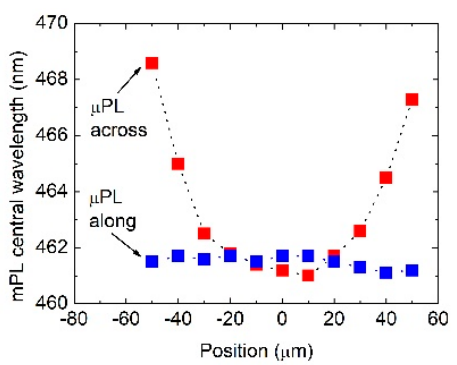

(b)

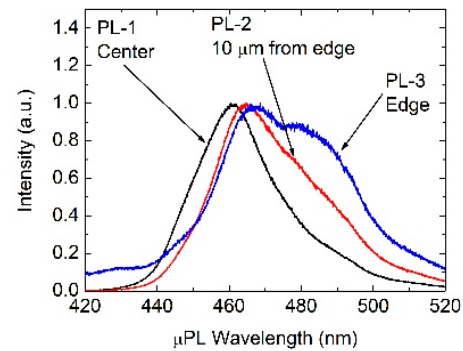

(c)

Figure 4. $\mu \mathrm{PL}$ of the InGaN/GaN structure. Stripe width $100 \mu \mathrm{m}$, stripe height $4 \mu \mathrm{m}$. (a) Measurement scheme with two scan lines across and along the stripe. (b) Wavelength across and along the stripe. (c) Spectra acquired at stripe center, $10 \mu \mathrm{m}$ from stripe edge, and at the stripe edge.

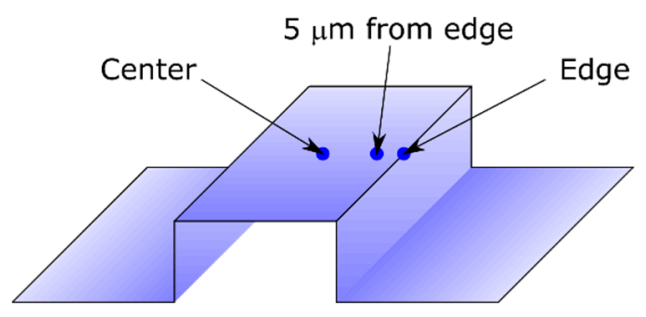

(a)

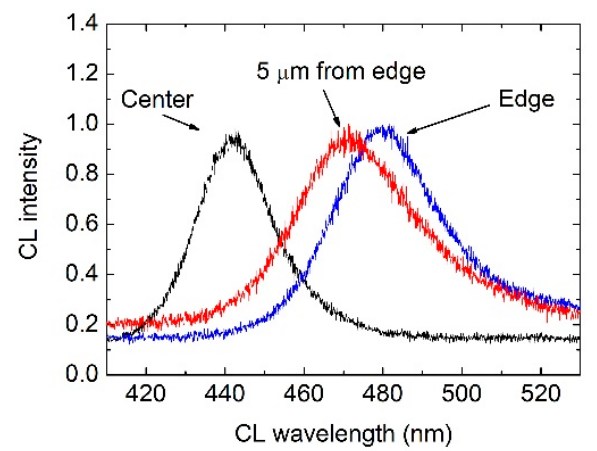

(b)

Figure 5. Cathodoluminescence (CL) of the InGaN/GaN structure. Stripe width was $50 \mu \mathrm{m}$. (a) Scheme of CL measurement with three characteristic points. (b) CL spectra acquired at stripe center, $5 \mu \mathrm{m}$ from the stripe edge, and at the stripe edge.

\subsection{Structural Properties}

Structure parameters, i.e., $\mathrm{QW}$ and $\mathrm{QB}$ thicknesses and $\mathrm{QW}$ indium content were examined independently in each of the patterned regions P5-P100 and in the reference region (see Figure 1 for a region layout explanation). Parameter values were obtained using Panalytical Epitaxy software with implemented dynamical theory of diffraction, by fitting calculated X-ray scans to measured ones, as shown in Figure 6a. It was found that QW thickness increased from 2 up to $3 \mathrm{~nm}$ when stripe width was decreased from 100 down to $5 \mu \mathrm{m}$, respectively, as shown in Figure 6b. Changes of QW/QB thickness is clearly seen in Figure 6a by shifts of the Pendelosung fringes towards (0002) reflection of GaN substrate. Similarly, QB thickness increased slightly from 8.2 up to $9 \mathrm{~nm}$. At the same time, indium composition in QWs increased from $19 \%$ on $100 \mu \mathrm{m}$ wide stripes up to $23 \%$ on $5 \mu \mathrm{m}$ wide stripes, as shown in Figure 6b. Of course, due to the composition pulling effect [32], on narrow stripes, the 1-QW and the 5-QW structures could differ. However, the observed wavelength variations with respect to the stripe width were similar for both structures. 


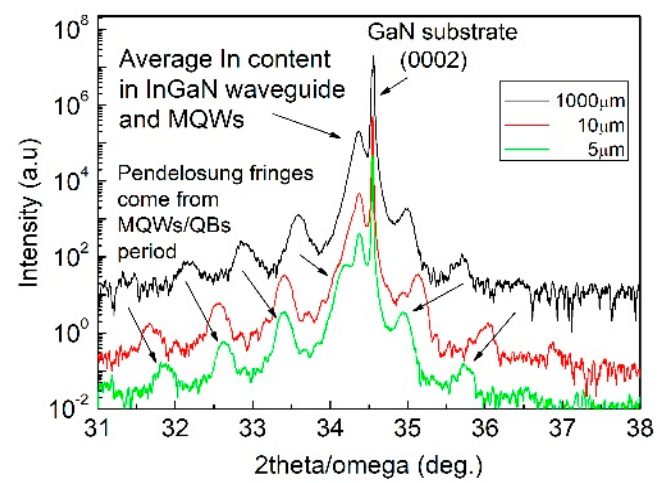

(a)

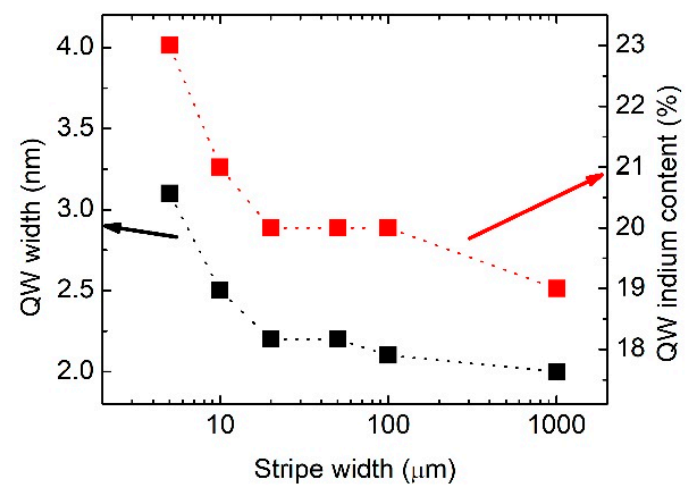

(b)

Figure 6. HR-XRD measurements of the InGaN/GaN MQW structure on substrate with $4 \mu \mathrm{m}$ high stripes; (a) 2theta-omega scans collected at $1000 \mu \mathrm{m}$ wide reference area, at $10 \mu \mathrm{m}$, and $5 \mu \mathrm{m}$ stripe regions (other regions not shown for picture clarity); (b) quantum well (QW) width and QW indium composition for different stripe widths obtained by fitting respective HR-XRD scans. The X-ray beam covers the whole width of each stripe.

\subsection{Influence of TMIn Flow and Temperature on Growth Rate on the Stripes}

To get more detail on the growth modes on the stripes, we examined the structure grown on patterned substrate with $4 \mu \mathrm{m}$ high stripes with a stylus profiler. We scanned all patterned regions of the structure in a direction perpendicular to the stripes, i.e., across the stripes, as shown in Figure 7a. It turned out that the structure height in the vicinity of the stripe edges is 100-150 nm larger than in the stripe center.

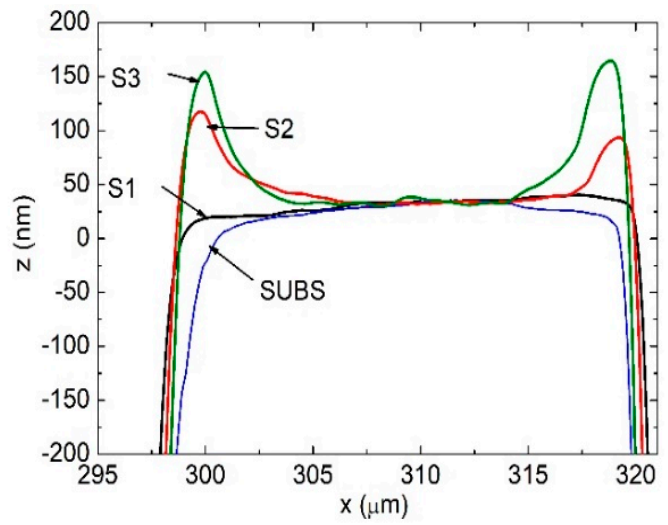

(a)

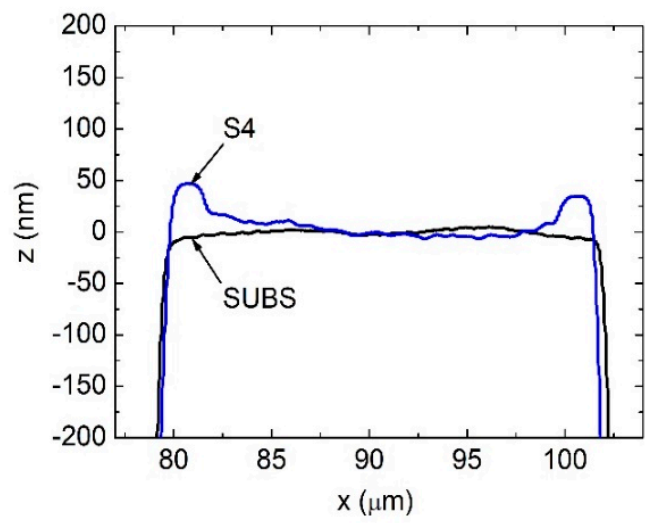

(b)

Figure 7. Surface profiles of InGaN/GaN structures grown on substrates with $20 \mu \mathrm{m}$ wide stripes. All profiles have been shifted in the z-direction to match each other's height in the center of the stripe. In (a) profile SUBS is for GaN substrate, S1-after growth of HT-GaN, S2 - after HT-GaN and InGaN, S3 after HT-GaN, InGaN, and 5QWs. In (b) profile SUBS is for GaN substrate and S4 after growth of HT-GaN + LT-GaN. See text and Table 2 for more details.

Table 2. Summary of samples used for surface profile characterization. QB: quantum barrier.

\begin{tabular}{ccc}
\hline Sample Name & Epitaxial Structure & Growth Temperature $\left({ }^{\circ} \mathbf{C}\right)$ \\
\hline SUBS & Patterned substrate & \\
S1 & HT-GaN & 1040 \\
S2 & HT-GaN + InGaN buffer & $1040+810$ \\
S3 & GaN + InGaN buffer $+5 \times(Q W+Q B)$ & $1040+810+760$ \\
S4 & HT-GaN + LT-GaN & $1040+810$ \\
\hline
\end{tabular}


Therefore, we decided to split our examined InGaN/GaN structure into parts, grow each of them separately on new patterned substrates, as shown in Table 2, and then check surface profile for each growth. Firstly, we measured the surface of patterned substrate in an identical manner (sample SUBS). Another profile was measured after growth of $0.5 \mu \mathrm{m}$ high-temperature GaN:Si (HT-GaN, $\mathrm{T}_{\mathrm{gr}}=1040{ }^{\circ} \mathrm{C}$, sample S1, see Table 2). Another profile was measured on epitaxial structure with $0.5 \mu \mathrm{m}$ HT-GaN GaN, followed by $170 \mathrm{~nm} \mathrm{InGaN,} \mathrm{T}_{\mathrm{gr}}=810^{\circ} \mathrm{C}$, sample S2-see Table 2 . The next profile was measured on epitaxial structure with five QW/QB pairs, sample S3-see Table 2. The results are shown in Figure 7a. Finally, we compared measurement of patterned substrate (sample SUBS), as shown in Table 2, to the sample which consisted of $0.5 \mu \mathrm{m}$ HT-GaN:Si followed by $170 \mathrm{~nm}$ thick LT-GaN (low temperature $\mathrm{GaN}, \mathrm{T}_{\mathrm{gr}}=810^{\circ} \mathrm{C}$ ), sample S4, Table 2. The results are shown in Figure $7 \mathrm{~b}$.

It turned out that the initial angle of the stripe sidewalls (70-80 to the c-plane) changed after growth of HT-GaN, because the exact-oriented, (11-22) facet has formed. An identical effect was observed in both reactors. That is why surface profiles of SUBS and S1 differ. Taking this fact into account, it can be seen that structure height at the stripe edges was larger only for GaN and InGaN layers grown at relatively low temperature, i.e., $810^{\circ} \mathrm{C}$ or lower. For HT-GaN, structure height on top of the stripe was the same as for the patterned substrate without any grown structure.

From HR-XRD results we learnt that the thickness of InGaN buffer, QWs, and QBs grown on top of the stripe were not lower but rather greater than the thickness of the same layers grown on the flat reference region. Nominally, the InGaN buffer thickness was $170 \mathrm{~nm}$. However, the height of the stripe measured near the stripe's edge is $100 \mathrm{~nm}$ larger than the height measured near the stripe's center. This means that the growth rate of the InGaN buffer close to the stripe edge must have been $60 \%$ larger than in the stripe center. Nominal thickness of five pairs of QW/QB was $50 \mathrm{~nm}$. Their surface profile close to the stripe edge extends by $\sim 50 \mathrm{~nm}$ over the InGaN buffer. It means that MQWs must have grown $100 \%$ faster close to the stripe edge compared to the center.

\subsection{Influence of the Stripe Height on Luminescence of In $G a N Q W s$.}

To investigate the influence of stripe height on morphology and optical properties of InGaN/GaN structures, we prepared another patterned substrate. It was nearly identical to the substrate described in Figure 1, but the stripe height was only $1 \mu \mathrm{m}$ instead of $4 \mu \mathrm{m}$. On this substrate we grew an InGaN/GaN epitaxial structure, identical to the one described in Section 2.2. It turned out that the luminescence wavelength shift in the case of $1 \mu \mathrm{m}$ high stripes was only $10 \mathrm{~nm}$ between the reference region and $5 \mu \mathrm{m}$ wide stripes, as shown in Figure 8a. Moreover, the effect of faster growth near the stripe edges was not observed for $1 \mu \mathrm{m}$ high stripes, as shown in Figure $8 \mathrm{~b}$.

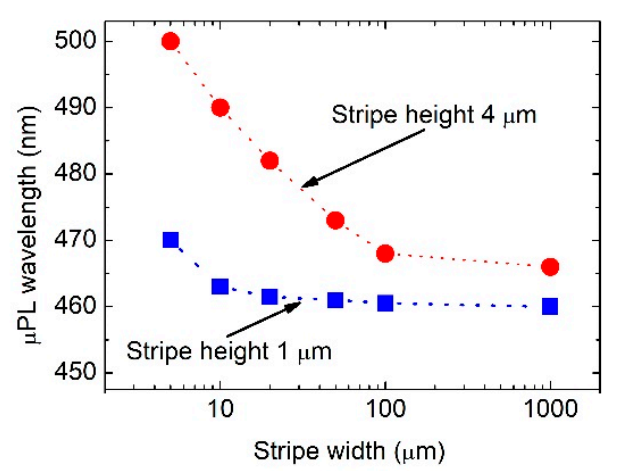

(a)

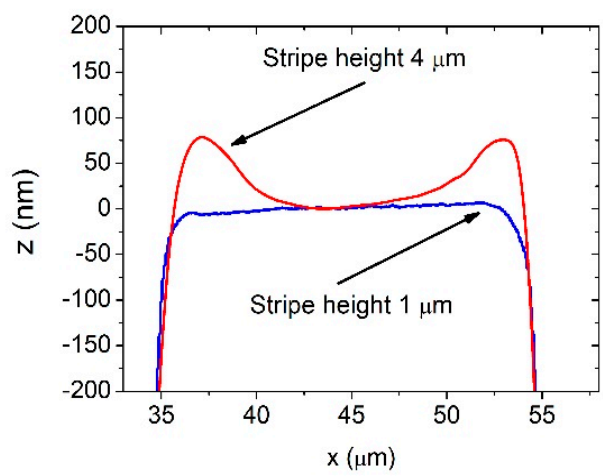

(b)

Figure 8. Luminescence (a) and surface profiles (b) of QW grown on substrates with stripes of different height. 


\section{Discussion}

The observed luminescence wavelength variations on different stripe widths may depend on the following factors and their combinations: (i) variations in quantum-confined Stark effect (QCSE) due to strain, (ii) variations in QW thickness, and (iii) variations in QW indium composition.

In order to assess the influence of elastic strains we performed numerical simulations of elastic energy per one InGaN molecule at the free surfaces of the stripe and the trench between the stripes, and the results are presented in Figure 9. For the simulations we used a standard finite-element method, and elastic constants of GaN and InGaN were taken from [13,33]. We performed strain simulations for the $20 \mu \mathrm{m}$ wide stripe and it turned out that in our design of patterned substrate, the influence of strain was limited to $2-3 \mu \mathrm{m}$ from the stripe edge, as shown in Figure 9. However, from $\mu$ PL data we see that luminescence wavelength shifts were observed not only near the stripe edges but on the whole $20 \mu \mathrm{m}$ wide stripe (wavelength shifts more than $20 \mathrm{~nm}$ at the stripe center, compared to the flat region of the substrate). Therefore, we conclude that mechanical strain could have an influence on wavelength shift only near the stripe edge and is probably not the main cause for the observed phenomena.
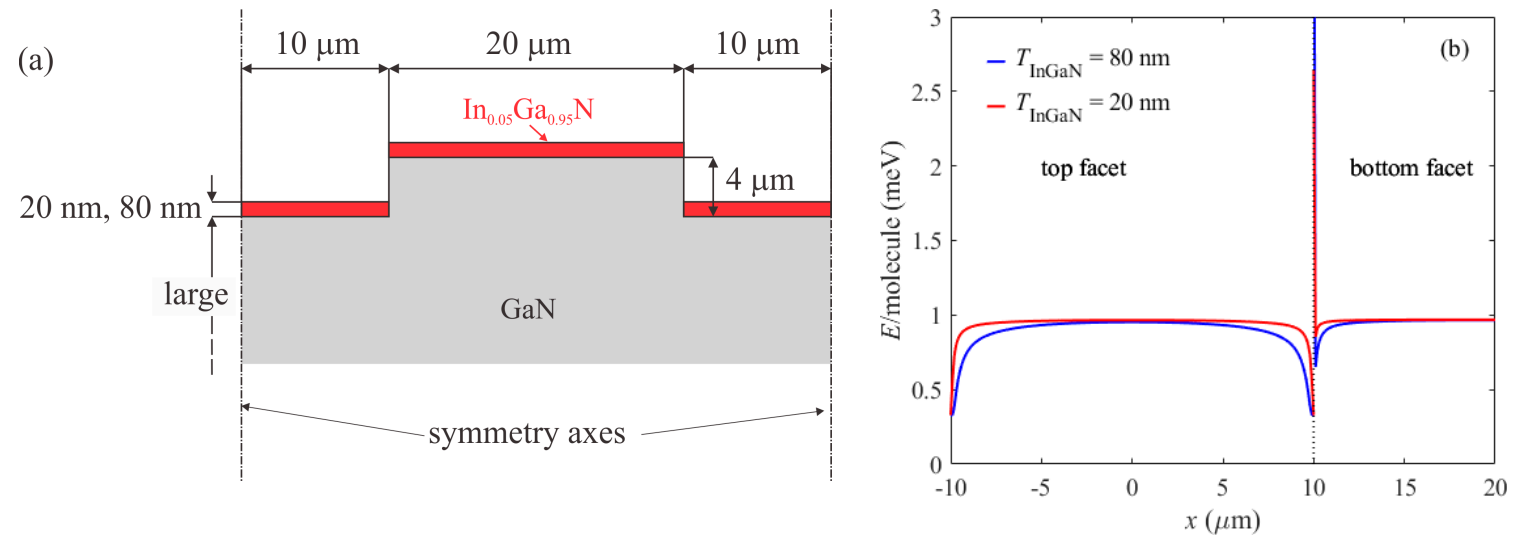

Figure 9. (a) Structure model used for the calculation of elastic energy. (b) Lateral profiles of the elastic energy per one InGaN molecule at the top and bottom InGaN surfaces.

Next, we tried to correlate QW thickness, growth rate, and indium composition on different stripes. According to HR-XRD measurements in the present work (Section 3.3), made on the 5QW structure, thicknesses of QWs and QBs on $5 \mu \mathrm{m}$ wide stripes were 3 and $9 \mathrm{~nm}$, respectively. However, on $100 \mu \mathrm{m}$ wide stripes the thicknesses were only 2 and $8 \mathrm{~nm}$, respectively. Indium composition in QWs was 23\% for the $5 \mu \mathrm{m}$ stripe and $19 \%$ for the $100 \mu \mathrm{m}$ one, as shown in Figure 6. Thus, the growth rate of the QW was $0.33 \AA / \mathrm{s}$ on $100 \mu \mathrm{m}$ stripes and $0.5 \AA / \mathrm{s}$ for $5 \mu \mathrm{m}$ ones.

The influence of the growth rate on indium composition has been investigated by Leszczyński et al. [29]. In that work, it was explained that incorporation of indium is larger at the higher growth rate because it is necessary to overbuild the indium atoms by gallium, otherwise it desorbs from the surface. Increased indium composition on narrow stripes could have been solely the result of faster growth. Hence, the increased $\mu \mathrm{PL}$ wavelength observed on narrow stripes can be explained by the joint effect of increased indium content and thicker QWs (thicker wells emit in longer wavelengths due to stronger QCSE).

Finally, we would like to know why the growth rate is higher on narrow stripes. As the observed effects occurred during MOVPE growth on patterned substrates, they should be discussed taking into account the following phenomena: (i) gas phase transport [34-36], (ii) gas phase reactions [37-39], (iii) gas phase diffusion [40-45], and (iv) surface diffusion [46-49].

Gas phase transport depends on the reactor design details. However, since we observed the effect in two very different reactors, we conclude that gas phase transport is not the most important factor here and we neglect it. 
Concerning gas phase reactions and gas phase diffusion, the presence of stripes (uneven surface) influences decomposition of the active species, their diffusion, and incorporation. The observed faster growth at the stripe edge would suggest that locally (edge area) the density of the active species participating in the growth is higher than in the stripe center or on the flat reference region. Because in our experiments the III-element sources were trimethylgallium and trimethylindium, the gas phase was dominated by their respective trimethyl (TM) and dimethyl (DM) species-DM resulting from TM decomposition. Although the DM lifetime is extremely short, it decomposes into monomethyl (MM), which diffuses on the surface with a given diffusion length, and in turn, is decomposed in adatoms, which also diffuse on the surface with their own diffusion lengths. All these species are affected by the presence of the stripes. The gas phase was preferentially decomposed at the stripe edges (possibly because of a higher temperature gradient [50]), thus inducing higher density of species (MM and adatoms) diffusing on the surface. As the stripe is higher, this effect becomes more pronounced.

Surface diffusion of MM species and adatoms will affect observed phenomena in a different way. The MM influences the distribution of adatoms on the surface, which consequently influences the local growth rate. Diffusion lengths of MM and adatoms can be estimated from the present experimental results. In this view, different indium compositions on different stripes, as shown in Figure $6 b$, would be an effect of MMIn diffusion (InGaN growth being In-limited), which is probably between 5 and $10 \mu \mathrm{m}$, since the composition was nearly constant for stripe widths larger than $20 \mu \mathrm{m}$. Similarly, diffusion of In adatoms is probably around 3-3.5 $\mu \mathrm{m}$ and it would explain faster growth observed up to 6-7 $\mu \mathrm{m}$ from the stripes' edge, as shown in Figure 7 . Thus, the adatom's diffusion explains the overgrowth at the stripe edge, and the diffusion of MM species explains why the concentration of indium is higher on small stripes.

On the other hand, such explanation is based on experiments where selective masks were used, and this is not the case of the present work. Therefore, we would like to present other arguments too.

We take into account the following effects: (i) gas-phase diffusion, (ii) alloy-pulling effect of indium due to mechanical strain, and (iii) kinetic processes on the surface. It 'is important to note that the faster growth at the edges is present only when growth temperature is relatively low $\left(820^{\circ} \mathrm{C}\right.$ rather than $\left.1000^{\circ} \mathrm{C}\right)$ and when the stripe height is relatively large $(4 \mu \mathrm{m}$ rather than $1 \mu \mathrm{m})$. Moreover, the effect is two times stronger for LT InGaN than for LT-GaN grown at the same conditions. There is no effect for structures grown at $1000{ }^{\circ} \mathrm{C}$, even on $4 \mu \mathrm{m}$ high stripes, and there is no effect for structures grown on $1 \mu \mathrm{m}$ high stripes even at $820^{\circ} \mathrm{C}$.

Certainly, gas phase diffusion would promote faster growth at the stripe edge. In the general case, based on a numerical solution of the diffusion equation, the distance which adatoms have to travel through the gas before they can adsorb at the crystal surface is the smallest at the edges, as in [40,51], as shown in Figure 8. Our own results obtained by a simple 2D, Monte Carlo simulation of the diffusion process confirms this effect.

Another point is that the faster growth of LT-InGaN compared to LT-GaN could be explained by the reduced alloy-pulling effect for indium at the 2-3 $\mu \mathrm{m}$ wide area close to the stripe edge [32]. Partial elastic relaxation of the structure near the edges would account for the low energy barrier for indium adatoms to incorporate into the crystal, as shown in Figure 9.

However, the most important here are kinetic processes at the growth surface. First, based on AFM data, we point out that the structure grows in the step-flow mode and thus the growth could be described in the framework of the Burton-Cabrera-Frank model [52], as shown in Figure 10. In this model adatoms adsorb on the growth surface from the gas phase and then diffuse, before they are caught by atomic steps and incorporate into the crystal. The second possibility is, however, that they desorb and return to the gas phase. The latter can happen mainly in high temperatures and low miscut conditions, i.e., when the atomic terrace width is comparable or larger than the adatom's surface diffusion length. 


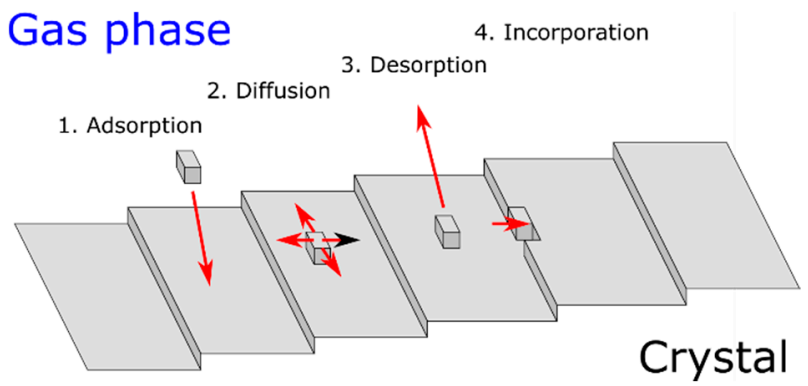

Figure 10. Different processes taken into account in the BCF model of crystal growth on vicinal surfaces.

Second, it is important to look at the structure morphology after growth. Judging from AFM, SEM, and STEM data, the top of the stripe is formed of (0001) plane which preserves the initial miscut of the substrate $\left(0.6^{\circ}\right.$ towards the $<1-100>$ direction), i.e., it contains regular atomic steps, with the average direction perpendicular to the stripe edge, separated by $20-30 \mathrm{~nm}$ wide terraces, as shown in Figure $2 \mathrm{~b}$ and inset to Figure 11a. On the contrary, the stripes' direction was precisely aligned $90 \pm 0.1^{\circ}$ to the easy-cleavage direction of $\mathrm{GaN},\langle 11-20\rangle$, and the sidewalls of the stripe (which are about $6 \mu \mathrm{m}$ wide for a $4 \mu \mathrm{m}$ stripe height) after growth become exact-oriented, low-index (11-22) planes, without any steps, as shown in Figure 11b,c. Hence, the density of sites where adatoms can attach is relatively high on the top of the stripe, compared to the sidewalls, where there are almost no such sites. It is important to note that on the (11-22) facet there was almost no growth, as shown in Figure 11c.

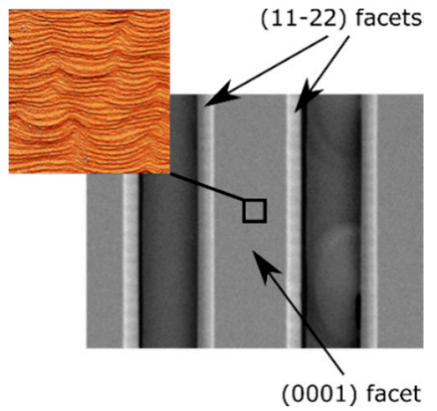

(a)

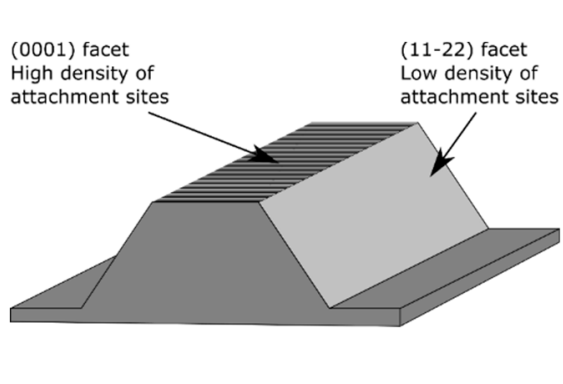

(b)

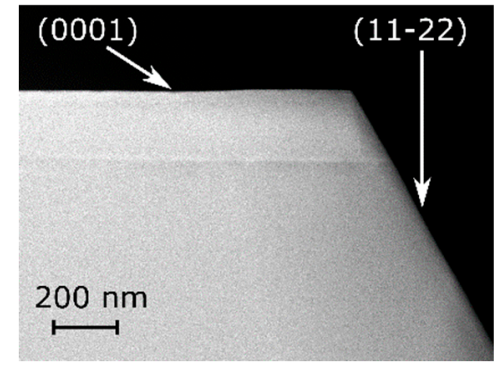

(c)

Figure 11. (a) Top-view SEM image of the structure grown on substrate with stripes. Image size $40 \times 30 \mu \mathrm{m}$. Inset: $2 \times 2 \mu \mathrm{m}$ AFM image acquired on the top of a $10 \mu \mathrm{m}$ wide stripe. (b) Schematic perspective view of the structure. The top of the stripe is formed of the vicinal (0001) plane and the sidewalls are exact-oriented (11-22) planes. (c) Cross-sectional scanning transmission electron microscopy (STEM) image.

Now let 'us assume that adatoms from the gas phase adsorb to both mentioned surfaces, i.e., the (0001) and (11-22) at equal rates. After adsorption they start to diffuse randomly on the surfaces. In the $\mathrm{BCF}$ theory, mean diffusion length of adatoms on the growth surface is expressed as

$$
\lambda=\mathrm{a} \exp \left[\left(\mathrm{W}_{\mathrm{s}}-\mathrm{U}_{\mathrm{s}}\right) / 2 \mathrm{kT}\right]
$$

where: a-lattice parameter in the growth plane, $\mathrm{U}_{\mathrm{s}}$ - energy barrier for adatom to move to the next stable position (process "2" from Figure 11), $\mathrm{W}_{\mathrm{s}}$ —energy barrier for adatom desorption (process " 3 " from Figure 10), T-absolute temperature.

Assuming that the growth is performed in nitrogen-rich conditions (which is almost always true in the case of MOVPE), growth kinetics will be governed by Ga and In diffusion on the surface. Mean diffusion lengths of these adatoms on (0001) and (11-22) facets have been estimated by Ueda and coworkers [42]. Those authors investigated InGaN grown by MOVPE on patterned substrate with a 
$\mathrm{SiO}_{2}$ mask. The growth temperature was not specified but we assume that it must have been adequate for InGaN growth, i.e., about $800{ }^{\circ} \mathrm{C}$. On (0001) they were 5.2 and $2.7 \mu \mathrm{m}$ for $\mathrm{Ga}$ and In, respectively, and on (11-22) they were 3.1 and $1.6 \mu \mathrm{m}$ for Ga and In, respectively. Hence, adatoms caught on the stripe top easily find an atomic step and attach (distance between adjacent atomic steps is $20-30 \mathrm{~nm}$ there). On the other hand, adatoms caught on the stripe sidewalls continue to diffuse until they reach the stripe edge because the surface is atomically flat and there are no atomic steps. Eventually they desorb back to the gas phase.

The balance between the caught and desorbed number of adatoms will depend on their mean diffusion length, which drops rapidly with temperature. At typical temperatures used for InGaN growth, the diffusion length is a few micrometers, i.e., of the order of the sidewall width [42]. Hence the (11-22) facets will catch adatoms and direct them towards the (0001) top facet where they can incorporate. The conclusion here is that the larger diffusion length, the faster growth near the stripe edge, i.e., fast growth will be promoted for low growth temperature. Faster growth at the stripe edges will also be promoted on taller stripes, because then the sidewall surface is larger. Since the (11-22) facet angle to the (0001) plane is 58.4 degrees [53], the (11-22) sidewall width $=1.6$ times the stripe height. This was confirmed by our results on 1 and $4 \mu \mathrm{m}$ tall stripes and by surface profiles of GaN grown at 800 and $1000^{\circ} \mathrm{C}$.

To sum up, faster growth of InGaN and its increased indium composition at the stripe edge could have been caused by all three factors-gas phase diffusion, the alloy-pulling effect, and kinetic processes-and the last factor is probably the most important.

\section{Conclusions}

GaN and InGaN layers, and InGaN/GaN MQW structures were grown on (0001) GaN substrates with stripes, at different growth temperatures, by MOVPE. The substrate stripes were parallel to the $<1-100>$ direction, they were $5-100 \mu \mathrm{m}$ wide, and typically $4 \mu \mathrm{m}$ high $(1 \mu \mathrm{m}$ on the test sample). There was no selective mask on the substrate. It was found that central wavelength of luminescence of MQW structures depend on the stripe width and height, and also growth temperature. It can be up to $40 \mathrm{~nm}$ larger on $5 \mu \mathrm{m}$ wide, $4 \mu \mathrm{m}$ tall stripes, compared to the flat area of the substrate (QW growth temperature $760^{\circ} \mathrm{C}$ ). We attribute this effect to faster growth on tall and narrow stripes. Faster growth promotes more effective indium incorporation, and of course QWs are thicker, which both account for the observed effect. Faster growth on tall and narrow stripes is caused mainly by kinetic processes at the growth surface. Notably, the initial substrate miscut angle and azimuth, and their relation to the direction of stripes is of primary importance for the observed effects. The reduced alloy-pulling effect for indium, due to partial elastic strain relaxation at the stripe edges, could also add to the observed effect.

Author Contributions: Conceptualization, M.S., E.G. and M.L.; funding acquisition, M.L., V.H. and H.A.; supervision, M.L., V.H. and H.A., writing-original draft preparation, M.S.; writing-review and editing, E.G.; investigation, M.S., E.G., S.G., G.T., R.C., V.H., A.R., S.N., and Z.L.; software, V.H. and M.S.

Funding: This work was supported by the Polish National Center for Research and Development, grant no V4-JAP/1/2016, Polish National Science Center, grant no 2015/17/B/ST5/02835 and JST (Japan Science and Technology Agency), Strategic International Collaborative Research Program, SICORP. V.H. acknowledges the support of the project NanoCent funded by the European Regional Development Fund (ERDF), project No CZ.02.1.01/0.0/0.0/15_003/0000485.

Conflicts of Interest: The authors declare no conflict of interest.

\section{References}

1. Rathman, D.D.; Silversmith, D.J.; Burns, J.A. Lateral epitaxial overgrowth of silicon on $\mathrm{SiO}_{2}$. J. Electrochem. Soc. 1982, 129, 2303-2306. [CrossRef] 
2. Tsaur, B.; McClelland, R.W.; Fan, J.C.C.; Gale, R.P.; Salerno, J.P.; Vojak, B.A.; Bozler, C.O. Low-dislocation-density GaAs epilayers grown on Ge-coated Si substrates by means of lateral epitaxial overgrowth. Appl. Phys. Lett. 1982, 41, 347-349. [CrossRef]

3. Gale, R.P.; McClelland, R.W.; Fan, J.C.C.; Bozler, C.O. Lateral epitaxial overgrowth of GaAs by organometallic chemical vapor deposition. Appl. Phys. Lett. 1982, 41, 545-547. [CrossRef]

4. Jastrzebski, L. Growth of electronic quality silicon over $\mathrm{SiO}_{2}$ by epitaxial lateral overgrowth technique. J. Electrochem. Soc. 1982, 129, 2645. [CrossRef]

5. Kato, Y.; Kitamura, S.; Hiramatsu, K.; Sawaki, N. Selective growth of wurtzite GaN and AlxGa1-xN on GaN/sapphire substrates by metalorganic vapor phase epitaxy. J. Cryst. Growth 1994, 144, 133-140. [CrossRef]

6. Usui, A.; Sunakawa, H.; Sakai, A.; Yamaguchi, A.A. Thick GaN epitaxial growth with low dislocation density by hydride vapor phase epitaxy. Jpn. J. Appl. Phys. 1997, 36, 899-902. [CrossRef]

7. Nakamura, S. First III-V-nitride-based violet laser diodes. J. Cryst. Growth 1997, 170, 11-15. [CrossRef]

8. Zheleva, T.S.; Nam, O.H.; Bremser, M.D.; Davis, R.F. Dislocation density reduction via lateral epitaxy in selectively grown GaN structures. Appl. Phys. Lett. 1997, 71, 2472-2474. [CrossRef]

9. Dadgar, A.; Bläsing, J.; Diez, A.; Alam, A.; Heuken, M.; Krost, A. Metalorganic chemical vapor phase epitaxy of Crack-Free GaN on Si (111) exceeding $1 \mu \mathrm{m}$ in thickness. Jpn. J. Appl. Phys. 2000, 39, 1183-1185. [CrossRef]

10. Gehrke, T.; Linthicum, K.J.; Preble, E.; Rajagopal, P.; Ronning, C.; Zorman, C.; Mehregany, M.; Davis, R.F. Pendeo-epitaxial growth of gallium nitride on silicon substrates. J. Electron. Mater. 2000, 29, 306-310. [CrossRef]

11. Oliver, R.A.; Briggs, G.A.D.; Kappers, M.J.; Humphreys, C.J.; Yasin, S.; Rice, J.H.; Smith, J.D.; Taylor, R.A. InGaN quantum dots grown by metalorganic vapor phase epitaxy employing a post-growth nitrogen anneal. Appl. Phys. Lett. 2003, 83, 755-757. [CrossRef]

12. Qian, F.; Li, Y.; Gradečak, S.; Park, H.G.; Dong, Y.; Ding, Y.; Wang, Z.L.; Lieber, C.M. Multi-quantum-well nanowire heterostructures for wavelength-controlled lasers. Nat. Mater. 2008, 7, 701-706. [CrossRef] [PubMed]

13. Morales, F.M.; Mánuel, J.M.; García, R.; Reuters, B.; Kalisch, H.; Vescan, A. Evaluation of interpolations of InN, AlN and GaN lattice and elastic constants for their ternary and quaternary alloys. J. Phys. D Appl. Phys. 2013, 46, 245502. [CrossRef]

14. Leszczynski, M.; Teisseyre, H.; Suski, T.; Grzegory, I.; Bockowski, M.; Jun, J.; Porowski, S.; Pakula, K.; Baranowski, J.M.; Foxon, C.T.; et al. Lattice parameters of gallium nitride. Appl. Phys. Lett. 1996, 69, 73-75. [CrossRef]

15. Yoshimoto, N.; Matsuoka, T.; Sasaki, T.; Katsui, A. Photoluminescence of InGaN films grown at high temperature by metalorganic vapor phase epitaxy. Appl. Phys. Lett. 1991, 59, 2251-2253. [CrossRef]

16. Keller, S.; Keller, B.P.; Kapolnek, D.; Mishra, U.K.; DenBaars, S.P.; Shmagin, I.K.; Kolbas, R.M.; Krishnankutty, S. Growth of bulk InGaN films and quantum wells by atmospheric pressure metalorganic chemical vapour deposition. J. Cryst. Growth 1997, 170, 349-352. [CrossRef]

17. Schenk, H.P.D.; de Mierry, P.; Laügt, M.; Omnès, F.; Leroux, M.; Beaumont, B.; Gibart, P. Indium incorporation above $800{ }^{\circ} \mathrm{C}$ during metalorganic vapor phase epitaxy of InGaN. Appl. Phys. Lett. 1999, 75, 2587-2589. [CrossRef]

18. Kaneta, A.; Funato, M.; Narukawa, Y.; Mukai, T.; Kawakami, Y. Direct correlation between nonradiative recombination centers and threading dislocations in InGaN quantum wells by near-field photoluminescence spectroscopy. Phys. Status Solidi Curr. Top. Solid State Phys. 2006, 3, 1897-1901. [CrossRef]

19. Nakamura, S. The Roles of Structural Imperfections in InGaN-Based Blue Light-Emitting Diodes and Laser Diodes. Science 1998, 281, 956-961. [CrossRef]

20. Jouvet, N.; Kappers, M.J.; Humphreys, C.J.; Oliver, R.A. The impact of substrate miscut on the morphology of InGaN epitaxial layers subjected to a growth interruption. J. Appl. Phys. 2013, 113, 063503. [CrossRef]

21. Lermer, T.; Pietzonka, I.; Avramescu, A.; Brüderl, G.; Müller, J.; Lutgen, S.; Strauss, U. Interdependency of surface morphology and wavelength fluctuations of indium-rich InGaN/GaN quantum wells. Phys. Status Solidi Appl. Mater. Sci. 2011, 208, 1199-1202. [CrossRef]

22. Dreyer, C.E.; Alkauskas, A.; Lyons, J.L.; Speck, J.S.; Van de Walle, C.G. Gallium vacancy complexes as a cause of Shockley-Read-Hall recombination in III-nitride light emitters. Appl. Phys. Lett. 2016, 108, 141101. [CrossRef] 
23. Ristić, J.; Calleja, E.; Fernández-Garrido, S.; Cerutti, L.; Trampert, A.; Jahn, U.; Ploog, K.H. On the mechanisms of spontaneous growth of III-nitride nanocolumns by plasma-assisted molecular beam epitaxy. J. Cryst. Growth 2008, 310, 4035-4045. [CrossRef]

24. Bengoechea-Encabo, A.; Albert, S.; Müller, M.; Xie, M.Y.; Veit, P.; Bertram, F.; Sanchez-Garcia, M.A.; Zúñiga-Pérez, J.; de Mierry, P.; Christen, J.; et al. Selective area growth of AlN/GaN nanocolumns on (0001) and (11-22) GaN/sapphire for semi-polar and non-polar AlN pseudo-templates. Nanotechnology 2017, 28, 365704. [CrossRef] [PubMed]

25. Fang, H.; Yang, Z.J.; Wang, Y.; Dai, T.; Sang, L.W.; Zhao, L.B.; Yu, T.J.; Zhang, G.Y. Analysis of mass transport mechanism in InGaN epitaxy on ridge shaped selective area growth GaN by metal organic chemical vapor deposition. J. Appl. Phys. 2008, 103, 014908. [CrossRef]

26. Dróżdż, P.A.; Sarzyński, M.; Domagała, J.Z.; Grzanka, E.; Grzanka, S.; Czernecki, R.; Marona, Ł.; Korona, K.P.; Suski, T. Monolithic cyan—violet InGaN/GaN LED array. Phys. Status Solidi 2017, 214, 1600815. [CrossRef]

27. Franssen, G.; Suski, T.; Kryśko, M.; Łucznik, B.; Grzegory, I.; Krukowski, S.; Khachapuridze, A.; Czernecki, R.; Grzanka, S.; Mensz, P.; et al. Influence of substrate misorientation on properties of InGaN layers grown on freestanding GaN. Phys. Status Solidi Curr. Top. Solid State Phys. 2008, 5, 1485-1487. [CrossRef]

28. Keller, S.; Keller, B.P.; Kapolnek, D.; Abare, A.C.; Masui, H.; Coldren, L.A.; Mishra, U.K.; Den Baars, S.P. Growth and characterization of bulk InGaN films and quantum wells. Appl. Phys. Lett. 1996, 68, 3147-3149. [CrossRef]

29. Leszczynski, M.; Czernecki, R.; Krukowski, S.; Krysko, M.; Targowski, G.; Prystawko, P.; Plesiewicz, J.; Perlin, P.; Suski, T. Indium incorporation into InGaN and InAlN layers grown by metalorganic vapor phase epitaxy. J. Cryst. Growth 2011, 318, 496-499. [CrossRef]

30. Czernecki, R.; Kret, S.; Kempisty, P.; Grzanka, E.; Plesiewicz, J.; Targowski, G.; Grzanka, S.; Bilska, M.; Smalc-Koziorowska, J.; Krukowski, S.; et al. Influence of hydrogen and TMIn on indium incorporation in MOVPE growth of InGaN layers. J. Cryst. Growth 2014, 402, 330-336. [CrossRef]

31. Nagamatsu, K.; Nitta, S.; Ye, Z.; Nagao, H.; Miki, S.; Honda, Y.; Amano, H. Decomposition of trimethylgallium and adduct formation in a metalorganic vapor phase epitaxy reactor analyzed by high-resolution gas monitoring system. Phys. Status Solidi 2017, 254, 1600737. [CrossRef]

32. Hiramatsu, K.; Kawaguchi, Y.; Shimizu, M.; Sawaki, N.; Zheleva, T.; Davis, R.F.; Tsuda, H.; Taki, W.; Kuwano, N.; Oki, K. The Composition Pulling Effect in MOVPE Grown InGaN on GaN and AlGaN and its TEM Characterization. MRS Internet J. Nitride Semicond. Res. 1997, 2, e6. [CrossRef]

33. Wright, A.F. Elastic properties of zinc-blende and wurtzite AIN, GaN, and InN. J. Appl. Phys. 1997, 82, $2833-2839$. [CrossRef]

34. Theodoropoulos, C.; Mountziaris, T.J.; Moffat, H.K.; Han, J. Design of gas inlets for the growth of gallium nitride by metalorganic vapor phase epitaxy. J. Cryst. Growth 2000, 217, 65-81. [CrossRef]

35. Harafuji, K.; Hasegawa, Y.; Ishibashi, A.; Tsujimura, A.; Kidoguchi, I.; Ban, Y.; Ohnaka, K. Complex flow and gas-phase reactions in a horizontal reactor for GaN metalorganic vapor phase epitaxy. Jpn. J. Appl. Phys. Part 1 Regul. Pap. Short Notes Rev. Pap. 2000, 39, 6180-6190. [CrossRef]

36. Momose, T.; Kamiya, T.; Suzuki, Y.; Ravasio, S.; Cavallotti, C.; Sugiyama, M.; Shimogaki, Y. Kinetic analysis of GaN-MOVPE via thickness profiles in the gas flow direction with systematically varied growth conditions. ECS J. Solid State Sci. Technol. 2016, 5, P164-P171. [CrossRef]

37. Sugiyama, M.; Yasukochi, S.; Shioda, T.; Shimogaki, Y.; Nakano, Y. Reaction kinetics of GaN Metal-Organic Vapor-Phase epitaxy analyzed by multi-scale profiles of growth rate. ECS 2009, 25, 507-512.

38. Sekiguchi, K.; Shirakawa, H.; Chokawa, K.; Araidai, M.; Kangawa, Y.; Kakimoto, K.; Shiraishi, K. Thermodynamic analysis of trimethylgallium decomposition during GaN metal organic vapor phase epitaxy. Jpn. J. Appl. Phys. 2018, 57, 04FJ03. [CrossRef]

39. Zhang, Z.; Fang, H.; Yao, Q.; Yan, H.; Gan, Z. Species transport and chemical reaction in a MOCVD reactor and their influence on the GaN growth uniformity. J. Cryst. Growth 2016, 454, 87-95. [CrossRef]

40. Tanikawa, T.; Honda, Y.; Yamaguchi, M.; Amano, H. Effect of lateral vapor phase diffusion during the selective growth of InGaN/GaN MQW on semipolar and nonpolar GaN stripes. Phys. Status Solidi Appl. Mater. Sci. 2011, 208, 1175-1178. [CrossRef]

41. Shioda, T.; Tomita, Y.; Sugiyama, M.; Shimogaki, Y.; Nakano, Y. Selective area metal-organic vapor phase epitaxy of nitride semiconductors for multicolor emission. IEEE J. Sel. Top. Quantum Electron. 2009, 15, 1053-1065. [CrossRef] 
42. Ueda, M.; Hayashi, K.; Kondou, T.; Funato, M.; Kawakami, Y.; Narukawa, Y.; Mukai, T. Mechanisms of metalorganic vapor phase epitaxy of InGaN quantum wells on GaN microfacet structures. Phys. Status Solidi Curr. Top. Solid State Phys. 2007, 4, 2826-2829.

43. Tanaka, A.; Chen, R.; Jungjohann, K.L.; Dayeh, S.A. Strong geometrical effects in submillimeter selective area growth and light extraction of GaN light emitting diodes on sapphire. Sci. Rep. 2015, 5, 1-12. [CrossRef] [PubMed]

44. Coltrin, M.E.; Willan, C.C.; Bartram, M.E.; Han, J.; Missert, N.; Crawford, M.H.; Baca, A.G. Transport, Growth Mechanisms, and Material Quality in GaN Epitaxial Lateral Overgrowth. MRS Proc. 1998, 537, G6.9. [CrossRef]

45. Mitchell, C.C.; Coltrin, M.E.; Han, J. Mass transport in the epitaxial lateral overgrowth of gallium nitride. J. Cryst. Growth 2001, 222, 144-153. [CrossRef]

46. Gotschke, T.; Schumann, T.; Limbach, F.; Stoica, T.; Calarco, R. Influence of the adatom diffusion on selective growth of GaN nanowire regular arrays. Appl. Phys. Lett. 2011, 98, 103102. [CrossRef]

47. Matsuda, Y.; Funato, M.; Kawakami, Y. Growth mechanism of Polar-Plane-Free Faceted InGaN quantum wells. IEICE Trans. Electron. 2018, 101, 532-536. [CrossRef]

48. So, B.M.; Youn, S.B.; Im, I.T. Growth rate and composition of InGaN during InGaN/GaN quantum wells selective area metal-organic vapor phase epitaxy considering surface diffusion. J. Ind. Eng. Chem. 2013, 19, 1747-1751. [CrossRef]

49. Wang, X.; Hartmann, J.; Mandl, M.; Mohajerani, M.S.; Wehmann, H.H.; Strassburg, M.; Waag, A. Growth kinetics and mass transport mechanisms of GaN columns by selective area metal organic vapor phase epitaxy. J. Appl. Phys. 2014, 115, 163104. [CrossRef]

50. Li, Z.; Jiang, Y.; Yu, T.; Yang, Z.; Tao, Y.; Jia, C.; Chen, Z.; Yang, Z.; Zhang, G. Analyses of surface temperatures on patterned sapphire substrate for the growth of $\mathrm{GaN}$ with metal organic chemical vapor deposition. Appl. Surf. Sci. 2011, 257, 8062-8066. [CrossRef]

51. Xiao, R.F.; Alexander, J.I.D.; Rosenberger, F. Microscopic-growth morphologies in binary systems. Phys. Rev. A 1992, 45, 571-574. [CrossRef]

52. Myers-Beaghton, A.K.; Vvedensky, D.D. Generalized Burton-Cabrera-Frank theory for growth and equilibration on stepped surfaces. Phys. Rev. A 1991, 44, 2457-2468. [CrossRef]

53. Scholz, F.; Meisch, T.; Caliebe, M.; Schörner, S.; Thonke, K.; Kirste, L.; Bauer, S.; Lazarev, S.; Baumbach, T. Growth and doping of semipolar GaN grown on patterned sapphire substrates. J. Cryst. Growth 2014, 405, 97-101. [CrossRef] 